\title{
A Microdeletion at 12q24.31 Can Mimic Beckwith-Wiedemann Syndrome Neonatally
}

\author{
E. Baple ${ }^{a} \quad$ R. Palmer ${ }^{\text {a }} \quad$ R.C.M. Hennekam ${ }^{\text {a-c }}$ \\ ${ }^{a}$ Department of Clinical Genetics, Great Ormond Street Hospital for Children; ${ }^{b}$ Clinical and Molecular Genetics \\ Unit, Institute of Child Health, UCL, London, UK, and ' Department of Pediatrics, Academic Medical Center, UVA, \\ Amsterdam, The Netherlands
}

\author{
Key Words \\ ACADS - Anxieties - Beckwith-Wiedemann syndrome • \\ Contiguous gene syndrome $\cdot$ Deletion 12q24.31 $\cdot$ HNF1A • \\ Macroglossia $\cdot$ Neonatal hyperinsulinism $\cdot$ Overgrowth . \\ P2RX7
}

\begin{abstract}
We report on a patient who was initially suspected to have Beckwith-Wiedemann syndrome because of recurrent neonatal hypoglycaemias, macroglossia and overgrowth, but in whom no 11 p15 abnormality could be found. Follow-up showed continued overgrowth and disturbed glucose homeostasis, a marked developmental delay, and severe behavioural problems especially caused by anxieties. Array comparative genomic hybridization analysis showed a de novo 12 q24.31 interstitial deletion, which was confirmed by fluorescence in situ hybridization. The deleted region contains amongst others: HNF1 homeobox A (HNF1A) which is important for the regulation of gene expression in the liver and involved in maturity-onset diabetes of the young type 3 and insulin resistance; acyl-CoA dehydrogenase short chain (ACADS) which encodes an enzyme important in mitochondrial fatty acid beta-oxidation and can cause shortchain acyl-CoA dehydrogenese (SCAD) deficiency, and purinergic receptor P2X7 (P2RX7) which encodes a ligand-gated
\end{abstract}

ion channel, and of which polymorphisms are found with increased frequency in patients with psychiatric disorders, especially anxieties. We conclude the present patient has a hitherto undescribed contiguous gene syndrome, which can initially resemble Beckwith-Wiedemann syndrome.

Copyright $\odot 2010$ S. Karger AG, Basel

Beckwith-Wiedemann syndrome (BWS) is a wellknown multiple congenital anomalies syndrome with (asymmetric) overgrowth, macroglossia, abdominal wall defects, neonatal hypoglycaemia, and an increased risk for embryonic tumours as major manifestations [Weksberg et al., 2005]. A variety of molecular mechanisms have been associated with BWS patients, consisting of mutations, deletions, or disturbed epigenetic regulations of genes located at 11p15 [Maher and Reik, 2000]. In 10$15 \%$ of individuals with BWS the cause remains unknown [Weksberg et al., 2005].

Here we report on a patient who was diagnosed in the neonatal period as having BWS. Initial cytogenetic and molecular investigations directed for $11 \mathrm{p} 15$ abnormalities gave negative results. Subsequent follow-up showed a development different from BWS, and recent re-evaluation allowed detection of a submicroscopic imbalance at chromosome $12 \mathrm{q} 24.31$.

\section{KARGER \\ Fax +4161306 1234 \\ E-Mail karger@karger.ch}

www.karger.com (c) 2010 S. Karger AG, Basel

1661-8769/10/0011-0042\$26.00/0

Accessible online at:

www.karger.com/msy
Dr. R.C.M. Hennekam

Clinical and Molecular Genetics Unit

Institute of Child Health

30 Guilford Street, London WC1N 1EH (UK)

Tel. +44 207905 2608, Fax +44 207905 2832, E-Mail r.hennekam@ich.ucl.ac.uk 

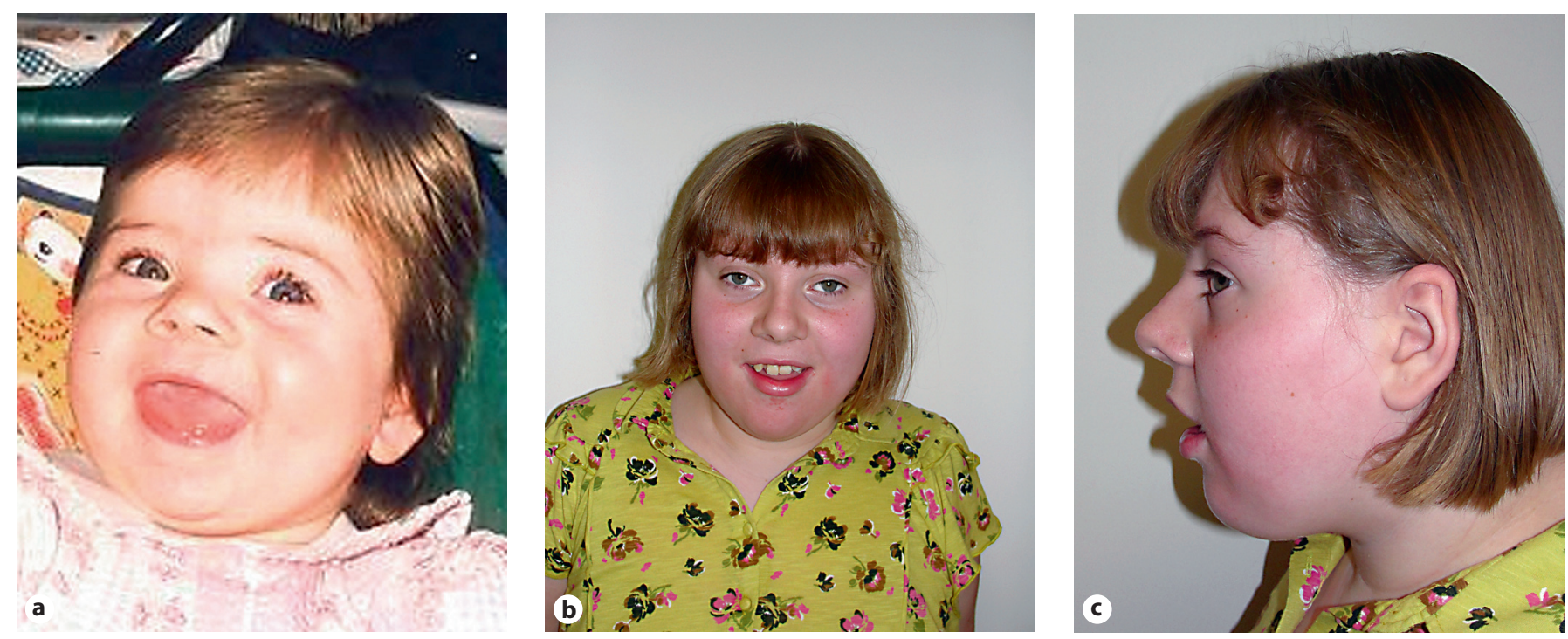

Fig. 1. a Proband at 9 months of age. Note the macroglossia, large mouth and full cheeks. b, c Proband at 13 years. Note upslanting palpebral fissures, broad nasal base, full cheeks, irregularly placed teeth, full and everted lower lip, and narrow but large ears with a thick helix.

\section{Case Report}

The proband was the first and only child of healthy, non-consanguineous parents. Family history was unremarkable. At 36 weeks of pregnancy the mother had a sudden unexplained weight gain. No clues for maternal diabetes were found. An emergency caesarean section was performed at term because of macrosomia. The girl weighted 4,094 g (90th-95th centile). At birth she had a large tongue which filled the mouth but did not cause respiratory problems. She did not have ear creases and her umbilicus was normal. She had immediate hypoglycaemias, which were found to be caused by hyperinsulinism. Management consisted of chlorthiazide, diazoxide and frequent nasogastric tube feedings, which remained needed during infancy to maintain a normoglycaemia. Weight gain was considerable: at 1 year her weight was $11.8 \mathrm{~kg}$ (98th centile). In early childhood gradually glucose control stabilized and she was successfully weaned off medication at the age of 4 years. The presence of a large tongue, recurrent neonatal hypoglycaemias, and rapid weight gain led to the suspicion of her having BWS (fig. 1a). Cytogenetic analysis showed a normal female karyotype, 46,XX, without evidence for an 11p15 duplication. A microsatellite marker mapping to the tyrosine hydroxylase locus was used to exclude mosaic 11p15 paternal isodisomy. A mutation screen of ABCC8 (OMIM 500509) gave normal results.

Developmental progress was very slow. She walked at 18 months and was able to use a few words at 2 years. She continued to be unsteady on her feet with a broad based gait. She developed marked anxieties, specifically about plants, small pieces of paper, loud noises and crowds which disturbed daily life significantly. A diagnosis of an autistic spectrum disorder was made at the age of 4 years. She continued to show overgrowth both in height and weight, despite dietary regimens. Urinary continence was achieved at 11 years. Except for significant constipation her general health has been good.
Re-examination at 11 years of age showed an overgrown girl, moderately to severely retarded, with a head circumference of $54.8 \mathrm{~cm}$ (75th centile), height of $157.5 \mathrm{~cm}$ (98th centile) and a weight of $91.5 \mathrm{~kg}$ (98th-99.6th centile). She had upslanted palpebral fissures, a broad nasal base, full cheeks, a highly arched palate, overcrowded teeth, full and everted lower lip, and large but narrow ears with a thick helix (fig. 1b, c). She had inverted nipples, a single small truncal café-au-lait spot, large hands and feet, mild tapering of the fingers, and short toes. The left 4 th finger was proximally implanted, and shortening of the 4 th metacarpal bone was confirmed radiologically.

\section{Molecular Analyses}

Microarray analysis using the BlueGnome CytoChip 2.01 (Genome Assembly NCBI 36) showed a cryptic interstitial deletion of the long arm of chromosome 12. The breakpoints of the deletion were within band 12q24.31. The deletion was between $1.58 \mathrm{Mb}$ and $2.53 \mathrm{Mb}$ in length and included the BACs RP11-18C24, RP11-44F24 and RP11-87C12 (fig. 2). The deletion was confirmed by fluorescent in situ hybridisation (FISH) using probes RP11$18 \mathrm{C} 24$ and RP11-87C12. Both parents gave a normal result by FISH using probe RP11-87C12, indicating that the deletion had arisen de novo.

Annotated genes in the deleted region are shown in figure 2 and include HNF1 homeobox A (HNF1A), acylcoenzyme A dehydrogenase, $\mathrm{C} 2$ to $\mathrm{C} 3$ short chain $(A C A D S)$, purinergic receptor $\mathrm{P} 2 \mathrm{X}$, ligand-gated ion 
Fig. 2. Ideogram showing the deleted region (between 1.58 and $2.53 \mathrm{Mb}$ in size) of $12 \mathrm{q} 24.31$ in the present proband, and the annotated genes in the region. BACs RP1118C24, RP11-44F24 and RP11-87C12 are deleted, the other BACs are present.

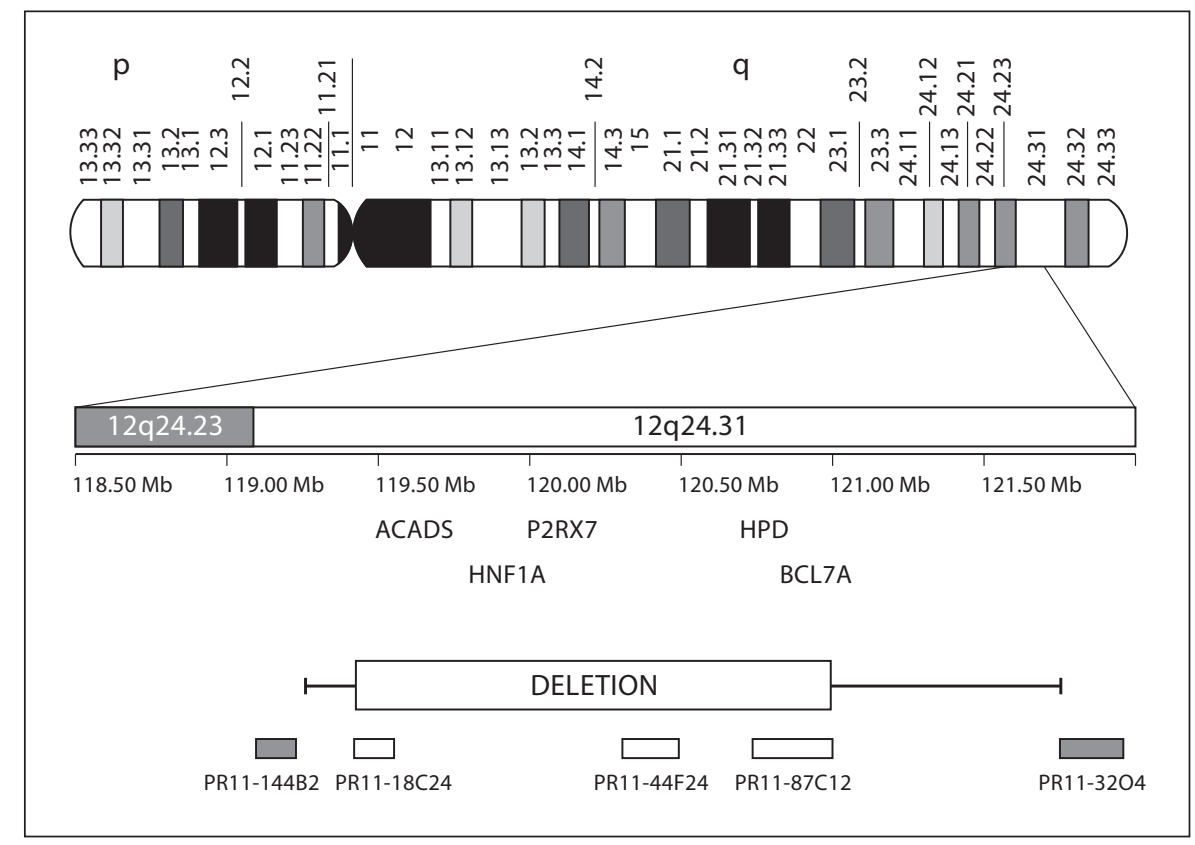

channel 7 (P2RX7), 4-hydroxyphenylpyruvate dioxygenase (HPD) and B-cell CLL/lymphoma 7A (BCL7A). The HNF1A gene has been shown to be important in glucose metabolism. Mutations in HNF1A (OMIM 142410) are associated with maturity-onset diabetes of the young type 3 (MODY3), susceptibility to insulin dependent diabetes mellitus, insulin resistance and hepatic adenomas.

ACADS (OMIM 606885) is a tetrameric mitochondrial flavoprotein similar to MCAD. Patients with homozygous mutations in $A C A D S$ present with variable features including hypoglycaemia, developmental delay, seizures, and behavioural difficulties. In some patients these symptoms are transient [Van Maldegem et al., 2006]. Polymorphisms in P2RX7 (OMIM 602566) are found with increased frequency in patients with chronic lymphatic leukaemia and psychiatric disorders such anxiety, phobias, depression and bipolar disorder [Kapoor, 2009]. HPD (OMIM 609695) is involved in the tyrosine catabolic pathway; heterozygous mutations are associated with Hawkinsinuria. Affected patients have failure to thrive and transient metabolic acidosis. Finally, variants in $B C L 7 A$ (OMIM 601406) have been shown to be a risk factor for the development of non-Hodgkin lymphoma [Morton et al., 2009].

\section{Discussion}

There have been only limited reports of patients with interstitial deletions involving band 12q24.31 and to our knowledge none with the same breakpoints or clinical features as our case. A male with a 12q24.31q24.33 deletion, developmental delay, tracheomalacia, genital abnormalities and minor cardiac defects was reported by Sathya and co-workers [1999]. Plotner et al. [2003] described a second cytogenetically visible case with microcephaly, moderate developmental delay, cardiac defects, growth failure, non-specific dysmorphic features and a 12q24.31q24.32 deletion. A review of terminal 12q deletions can be found elsewhere [Van Karnebeek, 2002].

The function of several of the genes within the deleted region seems to explain most of the features in the present proband well. The heterozygous deficiency of HNF1A may be responsible for the abnormal insulin levels in the neonatal period, and the unstable glucose homeostasis thereafter. It has been reported for patients with HNF4A mutations to have macrosomia at birth and hyperinsulinaemic hypoglycaemias in the neonatal period, evolving in decreased insulin secretion and diabetes later in life [Pearson et al., 2007]. This has not been reported for HNF1A though. The P2RX7 deletion might explain her unusual behavioural and social problems, and it is possible that the ACADS deficiency plays a role in her developmental delay, although this is much less certain. As 
such the present patient may be characterized as having a true contiguous gene syndrome. The deletion of several genes known to be involved in cancer development urged us to install a regular follow-up of the patient.

The initial resemblance of the proband to patients with Beckwith-Wiedemann syndrome is important for etiologic investigations in similar patients, and stresses the need to search for submicroscopic chromosome imbalances. The psychiatric profile in the proband and the deletion of $P 2 R X 7$ deserves further attention. Studies of series of patients with similar psychiatric illnesses for mutations in the receptor $P 2 R X 7$ are warranted. Possibly this may lead to better understanding of the aetiology and pathogenesis of such psychiatric disorders, and ultimately may lead to new therapeutic avenues.

\section{Acknowledgement}

We thank the patient and her family for their cooperation.

\section{References}

Kapoor S: Systemic and psychiatric disorders associated with polymorphism of the P2RX7 gene. Am J Med Genet Neuropsychiatr Genet 150B:597-598 (2009).

-Maher ER, Reik W: Beckwith-Wiedemann syndrome: imprinting in clusters revisited. J Clin Invest 105:247-252 (2000).

Morton LM, Purdue MP, Zheng T, Wang SS, Armstrong B, et al: Risk of non-Hodgkin lymphoma associated with germline variation in genes that regulate the cell cycle, apoptosis, and lymphocyte development. Cancer Epidemiol Biomarkers Prev 18:12591270 (2009).
Pearson ER, Boj SF, Steele AM, Barrett T, Stals K, et al: Macrosomia and hyperinsulinaemic hypoglycaemia in patients with heterozygous mutations in the HNF4A gene. PLoS Med 4:e118 (2007)

Plotner PL, Smith JL, Northrup H: Deletion 12q: A second patient with 12q24.31q24.32 deletion. Am J Med Genet 118A:350-352 (2003).

Sathya P, Tomkins DJ, Freeman V, Paes B, Nowaczyk MJM: De novo deletion 12q: report of a patient with $12 \mathrm{q} 24.31 \mathrm{q} 24.33$ deletion. Am J Med Genet 84:116-119 (1999).
Van Karnebeek CDM: Mental Retardation: Diagnostic Studies on Aetiology. Thesis, Amsterdam (2002).

-Van Maldegem BT, Duran M, Wanders R, Niezen-Konig K, Hogeveen M, et al: Clinical, biochemical, and genetic heterogeneity in short-chain acyl-coenzyme A dehydrogenase deficiency. JAMA 296:943-952 (2006).

Weksberg R, Shuman C, Smith AC: BeckwithWiedemann syndrome. Am J Med Genet 137C:12-23 (2005). 